\title{
Psychometric Properties and Convergent and Divergent Validity of the Portuguese Yale Food Addiction Scale 2.0 (P-YFAS 2.0)
}

\author{
Sónia Ferreira Gonçalves ( $\nabla$ sgoncalves@psi.uminho.pt ) \\ University of Minho https://orcid.org/0000-0003-1047-1503 \\ Célia Moreira \\ Universidade do Porto Faculdade de Ciencias \\ Bárbara Machado \\ Universidade Catolica Portuguesa \\ Beatriz Bastos \\ Universidade do Minho Escola de Psicologia \\ Ana Isabel Vieira \\ Universidade do Minho Escola de Psicologia
}

\section{Research Article}

Keywords: Food addiction, eating disordered behavior, emotion regulation, body investment

Posted Date: May 3rd, 2021

DOI: https://doi.org/10.21203/rs.3.rs-432446/v1

License: @ (i) This work is licensed under a Creative Commons Attribution 4.0 International License. Read Full License

Version of Record: A version of this preprint was published at Eating and Weight Disorders - Studies on Anorexia, Bulimia and Obesity on May 30th, 2021. See the published version at https://doi.org/10.1007/s40519-021-01218-0. 


\section{Abstract}

Purpose The construct of food addiction has been gaining increased attention as a research topic. Currently, the Yale Food Addiction Scale 2.0 is the only measure to operationalize the addictive-like eating behavior according to addiction criteria proposed by the Diagnostic and Statistical Manual of Mental Disorders. The present study aimed at examining the psychometric properties of the Portuguese version of the Yale Food Addiction Scale 2.0, as well as investigating the convergent and divergent validity between this scale and the following measures: Eating Disorders Examination Questionnaire, Body Investment Scale, and Difficulties in Emotion Regulation Scale. We also sought to explore the moderator role of difficulties in emotion regulation in the relationship between food addiction and binge eating. Methods: A sample of 302 female college students $\left(M_{a g e}=21.37, S D=3.24\right)$ completed selfreport measures.

Results Sixteen (5.3\%) participants were diagnosed as having food addiction. The confirmatory factor analysis suggested that the original onedimensional structure is adequate to represent the Portuguese Yale Food Addiction Scale 2.0. The symptom count scores of the scale were correlated with body mass index, eating disordered behavior, body investment and difficulties in emotion regulation. The severity level of the scale also discriminated the severity of eating disordered behaviors, body investment and difficulties in emotion regulation. Finally, the relationship between food addiction and binge eating was moderated by difficulties engaging in goal-directed behavior when experiencing negative emotions.

Conclusion The Portuguese version of the Yale Food Addiction Questionnaire 2.0 may be a useful tool to investigate food addiction.

\section{Introduction}

Similarities in biological, psychological, and behavioral factors implicated in addiction and disordered eating have led to the hypothesis that an addictive process may contribute to excess food consumption. The construct of food addiction (FA) has received increased clinical interest in recent years and describes a maladaptive form of eating that is thought to have similar patterns of neural activation that are also observed in substance use disorders (Gearhardt et al., 2011).

Few instruments have been used to identify addictive eating behavior. The most used tool to evaluate FA symptomatology is the Yale Food Addiction Scale (YFAS). This is a popular, widely used instrument and a standard measure for capturing addiction-like eating tendencies. The original version of YFAS was published by Gearhardt, Corbin, and Brownell (2009), but the fifth edition of the Diagnostic and Statistical Manual of Mental Disorders (DSM5; American Psychiatric Association, 2013) introduced important changes for the diagnosis of substance-related and addictive disorders and the authors created an updated version 7 years later, the YFAS 2.0 (Gearhardt, Corbin, \& Brownell, 2016). This newest version of YFAS included the combination of substance abuse and substance dependence, the exclusion of legal consequences diagnostic criterion, and the addition of craving (Hasin et al., 2013). According to the authors, this new version of YFAS captures the full range of symptoms comprising substance-related and addictive disorders (SRADs) considered in the DSM-5. Therefore, the development of the YFAS 2.0 allows for a more direct comparison of FA to other SRADs.

Research studies using the YFAS 2.0 have associated the prevalence of FA diagnosis with multiple factors such as age, sex or socioeconomic level, with women being more likely to experience FA, as well as populations from low socioeconomic status (Carr, Catak, Pejsa-Reitz, Saules, \& Gearhardt, 2017). In broad samples, the prevalence rates of FA diagnosis ranged between 3 to 20\% (Meule \& Gearhardt, 2019). Moreover, the prevalence of reported FA diagnoses is consistently greater in overweight and obese samples, compared to normal-weight or underweight individuals, and in samples with binge eating behavior. According to a recent review of studies using YFAS 2.0 (Meule \& Gearthardt, 2019), the highest prevalence rates of FA diagnoses were found in individuals with bulimia nervosa, followed by binge eating disorder, anorexia nervosa, subthreshold eating disorders, obesity, and unselected samples. YFAS 2.0 scores were also associated with other disordered eating behaviors, as well as with several comorbid mental disorders such as depression, anxiety disorders, posttraumatic stress disorder, and attention deficit hyperactivity disorder (Meule \& Gearthardt, 2019). Although the rates of FA may be lower in community samples compared to clinical samples, this form of eating emerges in the general population, including college students (Sengor \& Gezer, 2020; Wu, Zimmer, Munn-Chernoff, \& Baker, 2020). Furthermore, a recent study suggested that YFAS 2.0 is a good instrument to assess FA in both clinical and general populations (Manzoni et al., 2020). Therefore, it seems relevant to conduct further studies using YFAS 2.0 as an assessment tool of FA in community samples.

FA is usually positively associated with body mass index (BMI) and strongly linked with the presence of binge eating (Long, Blundell, \& Finlayson, 2015). Even so, in a recent study, a significant but moderate correlation was found between YFAS 2.0 symptom count and BMI, suggesting a nonlinear relation between these two variables (Manzoni et al., 2020). Additionally, the construct of FA can be considered within the context of impulsive behaviors and difficulties in emotion regulation, as assessed by the Difficulties in Emotion Regulation Scale (Gratz \& Roemer, 2004). This scale was developed to assess emotion dysregulation and it covers various difficulties, such as low awareness and clarity of emotions, non-acceptance of emotions, difficulties in the ability to control impulsive behaviors and to behave in accordance with goals when experiencing negative emotions, and limited use of appropriate emotion regulation strategies to modulate emotional responses (Gratz \& Roemer, 2004). Previously, a study evaluated the relationship between FA and difficulties in emotion regulation among women with eating disorders and non-suicidal self-injury (Carlson et al., 2018). Findings of this study suggested a shared aetiology between eating disorders, non-suicidal self-injury, and FA, explained possibly in part by emotion regulation deficits. Thus, FA may be associated with difficulties in emotion regulation, facilitating the engagement in maladaptive eating behaviors to cope with emotions, such as binge eating. Accordingly, in the study of Rossi, Gearhardt, Castelnuovo, and Mannarini (2020), FA was associated with emotional eating which may have led to experience negative feelings or cognitions, which in turn may have led to binge eating. So, it is possible that certain difficulties in emotion regulation 
exacerbate the relationship between FA and binge eating. Nevertheless, few studies to date have evaluated the relationship between these constructs and no research has examined difficulties in emotion regulation as a moderator of the association between FA and binge eating.

Moreover, the new version of YFAS has been validated in different languages, such as in German (Meule, Müller, Gearhardt, \& Blechert, 2017), Italian (Aloi et al., 2017; Manzoni et al., 2020), French (Brunault et al., 2017), Spanish (Granero et al, 2018), Japanese (Khine et al., 2019), Turkish (Sevinçer et al., 2014), Korean (Kim et al., 2019) and Arabic (Fawzi \& Fawzi, 2018). But to the best of our knowledge, only the first version of YFAS is available in Portuguese (Torres et al., 2017). Therefore, we aimed at assessing the psychometric properties and convergent and divergent validity of the Portuguese YFAS 2.0 in a non-clinical sample of Portuguese female college students. We also aimed at investigating the relationship between YFAS 2.0 and BMI, binge eating and other eating disordered behaviors, as assessed by Eating Disorders Examination Questionnaire (Fairburn \& Beglin, 2008), domains of the body investment of the Orbach and Mikulincer's Body Investment Scale (1998) and difficulties in emotion regulation. As we already mentioned, a study evaluated the relationship between FA and emotion regulation, and its findings suggested an aetiological-based explanation between them (cf. Carlson et al., 2018). So, this explanatory model needs more studies and replications, and given the significant overlap between FA and broader addictions, it is reasonable to assume that emotion regulation deficits may be a risk factor or correlate among individuals with FA. Additionally, FA has been associated with body image concerns (e.g., Meadows, Nolan, \& Higgs, 2017; Şanlier, Türközü, \& Toka, 2016) but the existing research is mainly focused on body image evaluation, and the role of other dimensions of body image, such as body investment, remains unclear. According to Orbach and Mikulincer (1998), body investment includes four domains: body image feelings and attitudes, comfort in physical touch, body care and body protection. A negative body investment is related to self-destructive tendencies, including eating disordered behaviors (Orbach \& Mikulincer, 1998). Thus, it is likely that a negative body investment may also be related to high FA, but no study to date has evaluated the relationship between these variables.

\section{Methods}

\section{Participants}

In this study, 302 female college students were recruited from a public Portuguese university. Ages ranged from 17 to 36 years $(M=21.37, S D=3.24)$. Mean BMI was $22.47(S D=4.85)$. Most participants were normal weight $(66.9 \% ; n=226)$ and few were underweight $(8 \% ; n=27)$, overweight $(9.5 \% ; n=$ $32)$ or obese $(5 \% ; n=17)$.

\section{Measures}

\section{Sociodemographic and clinical questionnaire}

This measure aims at collecting sociodemographic and clinical data of the participants, including age, gender, weight, and height.

\section{Portuguese Yale Food Addiction Scale 2.0 (P-YFAS 2.0)}

As the original version, the P-YFAS 2.0 assesses addiction-like eating behavior during the past 12 months. The scale consists of 35 items, which are scored on an eight-point scale ranging from never (score $=0$ ) to every day (score $=7$ ). The YFAS 2.0 provides two scoring options, one option that measures FA symptoms and another option that provides a FA diagnosis (Gearhardt et al., 2016). The 2.0 version evaluates 11 symptoms of FA: overeating (Criterion 1), desire to cut down (Criterion 2), time spent (Criterion 3), craving (Criterion 4), related impairment (work/school, family, social relationship) (Criteria 5-7), risky use (physically hazardous, detrimental physical/psychological consequences) (Criteria 8-9), tolerance (Criterion 10), and withdrawal (Criterion 11). Each of these 11 diagnostic criteria was considered fulfilled if one or more of the relevant questions for each criterion reached the threshold. A final symptom count score can be calculated by adding up all endorsed symptoms; thus, scores can range from 0 to 11 . Another score regards the severity level that is described according to the diagnostic thresholds for SRAD in DSM-5: mild FA (when 2-3 symptoms are present), moderate FA (when 4-5 symptoms have been recognized), and severe FA (when 6 symptoms are present). Every FA diagnosis requires the presence of the impairment or distress criteria.

\section{Eating Disorders Examination Questionnaire (EDE-Q)}

The Eating Disorder Examination Questionnaire (EDE-Q; Fairburn \& Beglin, 2008; Portuguese version Machado et al., 2014) is a self-report measure that assesses the frequency and severity of eating disorder symptoms over the past 28 days. The EDE-Q includes 28 items divided into four subscales: restraint, eating concern, shape concern, and weight concern. In the present study, Cronbach's alpha of the EDE-Q total score was .95, and Cronbach's alphas of the EDE-Q subscales were as follows: restraint, $a=.84$; eating concern, $a=.79$; shape concern, $a=.88$; and weight concern, $a=.84$.

\section{Body Investment Scale (BIS)}

The Body Investment Scale (BIS; Orbach \& Mikulincer, 1998; Portuguese version Vieira, Fernandes, Machado, \& Gonçalves, 2020) taps a person's emotional investment in his or her body and includes subscales relating to feelings and attitudes about the body, body care, body protection, and comfort in physical touch. The self-administered BIS contains four subscales with six items each: body image includes items exploring feelings and attitudes about one's body; body touch consists of statements about one's comfort with physical touch; body care includes items concerning body care; and body protection consists of items about desire for protection of one's body. Responses range from 1 (/ do not agree at all) to 5 (strongly agree), with higher scores indicating more positive responses about the body. In the present study, we obtained the following Cronbach's alphas: body image feeling and attitudes, $a=.94$; body touch, $a=.86$; body care, $a=.72$; and body protection, $a=.68$. 


\section{Difficulties in Emotion Regulation Scale (DERS)}

The Difficulties in Emotion Regulation Scale (DERS; Gratz \& Roemer, 2004; Portuguese version Coutinho, Ribeiro, Ferreira, \& Dias, 2010) is a self-report measure that assesses difficulties in emotion regulation. It contains 36 items rated on a 5-point scale across six dimensions of emotion regulation that include the following: limited access to emotion regulation strategies (Strategies); non-acceptance of emotional responses (Non-acceptance); lack of emotional awareness (Awareness); impulse control difficulties (Impulses); difficulties engaging in goal-directed behavior (Goals); and lack of emotional clarity (Clarity). It is possible to obtain a total score (adding all the 36 items) and a score for each subscale. Higher scores indicate greater difficulties in emotion regulation. In the present study, we obtained the following Cronbach's alphas for the subscales of strategies, $a=.88$, non-acceptance, $a=.89$, awareness, $a=.82$, impulses, $a=.91$, goals, $a=.89$, and clarity, $a=.88$. Cronbach's alpha for the DERS total score was .95 .

\section{Procedure}

For the development of the P-YFAS 2.0, the original English YFAS 2.0 was translated into Portuguese and back-translated into English.

The present research was approved by the Social Science's Ethics Committee of University of Minho. An informant consent with a brief explanation of the main aims of the study was provided to all participants. The security and confidentiality of the data was guaranteed.

\section{Statistical Analysis}

A confirmatory factor analysis (CFA) for dichotomous data was conducted using the R statistical environment (RStudio, version 3.6.2; R Development Core Team, 2019), through lavaan (Rosseel, 2012) and semTools (Jorgensen, Pornprasertmanit, Schoemann, \& Rosseel, 2018) to examine whether the 11 P-YFAS 2.0 symptoms had an underlying one-factorial structure. $R$ is a programming environment where statistical techniques are implemented. The capabilities of $R$ are extended through libraries containing collections of codes and functions with specific statistical purposes, the $R$ packages. For example, the lavaan package provides a variety of functions that allow to fit latent variable models, including factor analysis, structural equation modeling and latent growth curve models (Rosseel, 2012). The semTools package provides useful tools for structural equation modeling.

Following the procedure implemented in the original YFAS 2.0, factor structure and internal consistency of the P-YFAS 2.0 were examined at the symptoms and not at the items level (Gearhardt et al., 2016; Meule et al., 2017). Items assessing impairment or distress were not included in this analysis as they reflect clinical significance of the full syndrome (i.e., FA) rather than being indicators of individual criteria (Gearhardt et al., 2016; Meule et al., 2017). The comparative fit index (CFI), Tucker-Lewis index (TLI), weighted-root-mean-square residual (WRMR) and the root-mean-square error of approximation (RMSEA) were used to evaluate the model. The classic statistic $\chi^{2}$ of the model was also reported along with the model degrees of freedom $(d f)$, although it was not used as a goodness of fit measure as it is known that both large sample sizes and nonnormal data inflate this statistic (Brown, 2015; West, Taylor, \& Wu, 2012).

Internal consistency of the P-YFAS 2.0 symptoms was evaluated with Cronbach's alpha, McDonald's omega, and AVE (Average Variance Extracted; Fornell \& Larcker, 1981). We used the weighted least squares means and variance adjusted (WLSMV) estimator, which is recommended for categorical data and, in particular, for binary items (Brown, 2015). This is a robust variant of DWLS estimator, which uses the diagonal of the weight matrix (DWLS) to estimate the model parameters and uses the full weight matrix to correct the standard errors and to compute the test statistic.

All the remaining analyses were conducted on IBM® SPSS ${ }^{\circledR}$ (Statistical Package for the Social Sciences). That is, for the EDE-Q, DERS and BIS, internal consistency was examined by computing Cronbach's alphas. To assess the sociodemographic and clinical characteristics of the sample and the frequency of FA, descriptive statistics were conducted. Convergent and discriminant validity between the YFAS 2.0, EDE-Q, BIS and DERS was examined with analysis of variance (ANOVA) and Spearman's rank correlation. Following the procedure of Gearhardt et al. (2016), the scores of these questionnaires were compared according to the severity levels of YFAS 2.0.

To analyze whether the effect of FA (symptom count score; $X$ ) on frequency of binge eating episodes $(Y$ ) is moderated by difficulties in emotion regulation (total score and six domains of the DERS; $W$ ), we examined simple linear moderation models through a regression-based bootstrapping procedure - 5000 bootstrap samples and 95\% confidence intervals (Cls) - using the PROCESS macro for SPSS (Hayes, 2013). According to Hayes (2013), $W$ is moderator of the effect of $X$ on $Y$ if $X$ and $W$ interact in their influence on $Y$. The interaction term or product of $X$ and $W$ is estimated by the PROCESS and it is included in the regression model of $Y$ from $X$ and $W\left(b_{3}\right)$. This regression coefficient quantifies how the effect of $X$ on $Y$ changes as $W$ changes by one unit, and moderation is shown up by a significant interaction effect (Field, 2013; Hayes, 2013). Moreover, the regression model includes conditional effects that estimate the effect of $X$ when $W=0\left(b_{1}\right)$ and the effect of $W$ when $X=0\left(b_{2}\right)$. A $p<.05$ was considered statistically significant.

\section{Results}

\section{Frequency of food addiction}

Sixteen participants (5.3\%) from the sample resulted positive for at least two symptoms of the scale with clinically significant/impairment or distress. Regarding to the severity level, $3(1 \%)$ received a mild FA diagnosis, $5(1.7 \%)$ receive a moderate FA diagnosis and $8(2.6 \%)$ received a severe FA diagnosis.

\section{Symptom factor structure and reliability}


The CFI (.970), the TLI (.962), the WRMR (1.070), the RMSEA (.058), the 90\% confidence interval of RMSEA (.040-.075) and probability RMSEA (.227) suggested good fit for the one-factor model. Besides, we observed: $\chi^{2}=87.818, d f=44, p<.001$. All criteria had factor loadings for the single factor of .71 or higher (Table 1). The internal consistency measures of the model suggested good internal consistency: Cronbach's $a=.95 ;$ McDonald's $\omega=.90$; $\mathrm{AVE}=.68$.

Table 1 shows the frequencies of participants who met FA criteria. These indicated that the distribution was not normal.

\section{Convergent and divergent validity}

As displayed in Table 2, the YFAS 2.0 symptom count scores were significantly correlated with BMI, eating disordered behavior, difficulties in emotion regulation and body investment (ranging from .15 to .53). ANOVA results (Table 3) show that the severity level of YFAS 2.0 successfully discriminated the severity of eating-related constructs, body investment and difficulties in emotion regulation.

\section{The moderation of difficulties in emotion regulation in the relationship between food addiction and binge eating}

We analyzed simple linear moderation models for the total score and each of the six subscales of the DERS: limited access to emotion regulation strategies, non-acceptance of emotional responses, lack of emotional awareness, impulse control difficulties, difficulties engaging in goal-directed behavior and lack of emotional clarity. Except for difficulties engaging in goal-directed behavior when experiencing negative emotions, the models with the total score $(b=.003,95 \% \mathrm{Cl}[-.005, .012], t=.825, p=.410)$ and remaining DERS subscales did not show significant moderating effects: limited access to emotion regulation strategies $(b=.025,95 \% \mathrm{Cl}[-.002, .053], t=1.835, p=.068)$; non-acceptance of emotional responses $(b=.005,95 \% \mathrm{Cl}$ $[-.024, .034], t=.331, p=.741)$, lack of emotional awareness $(b=.002,95 \% \mathrm{Cl}[-.042, .045], t=.067, p=.947)$; impulse control difficulties $(b=-.030,95 \%$ $\mathrm{Cl}[-.067, .008], t=-1.533, p=.126)$; and lack of emotional clarity $(b=-.0003,95 \% \mathrm{Cl}[-.045, .045], t=-.012, p=.991)$. Therefore, we will present the model in which the moderator variable $(W)$ was difficulties engaging in goal-directed behavior when experiencing negative emotions.

As can be seen in the Table 4 and Figure 1, the effect of the interaction term $(X W)$ was positive and significant, $b=.075,95 \% \mathrm{Cl}[.030, .121], t=3.266, p=$ .001 , indicating that the relationship between FA and frequency of binge eating episodes is moderated by difficulties engaging in goal-directed behavior when experiencing negative emotions. Specifically, there is a significant positive relationship between FA and frequency of binge eating episodes at low

$(b=.566,95 \% \mathrm{Cl}[.191, .940], t=2.973, p=.003)$, medium $(b=.926,95 \% \mathrm{Cl}[.707,1.144], t=8.340, p<.001)$ and high $(b=1.286,95 \% \mathrm{Cl}[1.064,1.508], t=$ $11.401, p<.001$ ) levels of difficulties engaging in goal-directed behavior (Figure 2).

\section{Discussion}

The construct of FA postulates that certain foods (usually highly processed, highly palatable, and highly caloric foods) may have an addictive potential (Gearhardt et al., 2016). However, few measures exist to evaluate FA and, according to the authors of the YFAS 2.0, the development of this scale has provided an updated assessment tool to support continued investigation into the FA domain. Therefore, and considering that only the first version of YFAS was available in Portuguese, the main aim of this study was to evaluate the psychometric properties of P-YFAS 2.0 and to investigate possible associations of this scale with disordered eating behaviors, body investment and difficulties in emotion regulation.

This study confirmed the adequate psychometric properties of the Portuguese YFAS 2.0, suggesting the suitability of the original one-dimensional structure of YFAS 2.0. Thus, the Portuguese version of the YFAS 2.0 may be a useful tool to investigate FA, especially among college students. Sixteen participants (5.3\%) of our total sample were diagnosed with FA according to the YFAS 2.0. In a recent review of studies (Meule \& Gearhardt, 2019) using YFAS 2.0, the prevalence rates of FA diagnosis ranged between 3 to $20 \%$. In a similar sample of Italian college students, the prevalence of YFAS 2.0 diagnosis was $5.5 \%$ in females and $0.8 \%$ in males (Aloi et al., 2017). Regarding the frequency of FA diagnosis, our results also showed that severe FA diagnoses were more common than mild or moderate diagnoses. Additionally, these findings were consistent with the English (Gearhard et al., 2016), German (Meule et al., 2017) and Italian versions (Aloi et al., 2017).

Our results showed a significant but small positive association between YFAS 2.0 symptom count scores and BMI, as well as positive associations between FA and eating disordered behavior and binge eating episodes. In another study, Horsager, Færk, Lauritsen, and Østergaard (2021) found that participants with moderate and severe YFAS scores and comorbid mental disorders had elevated BMI. These results support the conclusion that those meeting an FA diagnosis are more likely to be overweight. For instance, Pedram et al. (2013) reported that 88.6\% of the so-called food addicts were overweight/obese. However, some studies (e.g., Burgess, Turan, Lokken, Morse, \& Boggiano, 2014) did not found an association between BMI and FA after controlling binge eating. In line with our results, in the study of Manzoni et al. (2020), there was a significant but moderate correlation between YFAS 2.0 symptom count and BMI, suggesting a nonlinear relation between these two variables. Therefore, more studies are needed to better understand the association between FA and BMI.

Yet, the association between FA and other disordered eating behaviors such as binge eating seems to be more consistent. Indeed, according to a recent review by Meule and Geardardt (2019), YFAS 2.0 scores were strongly associated with binge eating symptoms and frequency. However, in the same review, these authors concluded that the relationship between FA and other disorder symptoms, such as eating, weight and shape concerns are of moderate magnitude.

Our study is the first one, as far as we know, to investigate the association between FA and body investment. Results showed a negative significant association between FA and positive feelings and attitudes about the body, body care, body protection, and comfort in physical touch. Previous studies

Page 5/11 
(cf., Meule \& Gearhardt, 2019) reported correlations between FA and higher stress, hopelessness, and even suicidality and non-suicidal self-injury. The authors also found the same pattern between FA and the presence of disorders such as depression, anxiety, and posttraumatic stress disorder. Considering that body investment refers to the cognitive, behavioral, and emotional importance of the body in one's self-evaluation (Marco et al., 2017), we can speculate that lower body investment may be related to these above-mentioned symptoms and clinical disorders, and they may also be interconnected to emotion regulation deficits. Therefore, the results of our study highlight the relevance of evaluating, promoting, and intervening in body investment domains.

Our findings showed that the relationship between FA and binge eating is moderated by difficulties engaging in goal-directed behavior when experiencing negative emotions. The remaining dimensions of emotion dysregulation and the DERS total score did not play a moderating role in that relationship. As already described, the relationship between FA and binge eating is well supported by previous findings (Long et al., 2015; Meule \& Geardhart, 2019). However, few studies until now have evaluated the association between FA, binge eating, and difficulties in emotion regulation. Nevertheless, understanding FA as an addiction process may lead us to conceptualize this maladaptive form of eating as a regulatory strategy to deal with emotions. In this sense, FA may be understood as an inappropriate response to deal with negative emotions also meet in community samples. Moreover, given the moderating role of difficulties engaging in goal-directed behavior when experiencing negative emotions in the relationship between FA and binge eating, interventions targeting emotion regulation and effective problem solving strategies may be useful in the cessation or decreasing of FA and binge eating.

Although the current study provides important evidence regarding the psychometric properties and convergent and divergent validity of the Portuguese version of the YFAS 2.0, there are some limitations that should be considered. First, the use of self-report measures to evaluate complex constructs such as FA, disordered eating, body investment, or emotion regulation. Second, the cross-sectional nature of the study and the use of a female sample. So, results should not be generalized to all populations. Future research should use a Portuguese clinical sample with overweight/obese and/or eating disorders patients and a prospective design.

In sum, the P-YFAS 2.0 exhibited adequate internal reliability and showed good convergent validity with measures of similar constructs and good divergent validity relative to related but dissimilar constructs. We hope that our results contribute to better understand FA and to validate the Portuguese version of the YFAS 2.0, providing a useful tool to measure addictive-like eating behavior in community, clinical and research contexts.

\section{What is already known on this subject?}

FA has received increased attention over the last years. The YFAS 2.0 is the only measure to evaluate FA, according to the DSM-5 criteria. Since this scale has not been validated to the Portuguese population, we aimed at assessing the psychometric properties and convergent and divergent validity of the Portuguese version of YFAS 2.0. FA has also been associated with binge eating and higher BMI. Moreover, FA can underlie emotion dysregulation, facilitating the engagement in binge eating to cope with emotions. Possibly, certain difficulties in emotion regulation exacerbate the relationship between FA and binge eating. However, no research has examined the presence of difficulties in emotion regulation as a moderator of the association between these variables. In addition, the role of body image investment in FA remains unclear. Therefore, it is relevant to investigate correlates of YFAS, namely disordered eating behaviors, body investment and difficulties in emotion regulation and how they could represent identifiable targets for initiatives aimed at reducing FA.

\section{What does this study add?}

This study confirmed the adequate psychometric properties of the Portuguese YFAS 2.0. Thus, the Portuguese version of the YFAS 2.0 may be a useful tool to investigate FA, especially among college students. Given the moderating role of difficulties engaging in goal-directed behavior when experiencing negative emotions in the relationship between FA and binge eating, interventions targeting emotion regulation and effective problem solving strategies may be useful in the cessation or decreasing of FA and binge eating. FA also appeared negatively associated with the dimensions of the body investment. Therefore, it is relevant to consider the body investment as an assessment and intervention target among individuals with FA.

\section{References}

Aloi, M., Rania, M., Muñoz, R. C. R., Murcia, S. J., Fernández-Aranda, F., De Fazio, P., \& Segura-Garcia, C. (2017). Validation of the Italian version of the Yale Food Addiction Scale 2.0 (I-YFAS 2.0) in a sample of undergraduate students. Eating and Weight Disorders-Studies on Anorexia, Bulimia and Obesity, 22(3), 527-533.

American Psychiatric Association (2013). Diagnostic and statistical manual of mental disorders (5th ed.). Washington, DC: Author.

Brown, T. A. (2015). Confirmatory Factor Analysis for Applied Research (2nd ed.). New York: The Guilford Press.

Brunault, P., Courtois, R., Gearhardt, A. N., Gaillard, P., Journiac, K., Cathelain, S., ... \& Ballon, N. (2017). Validation of the French version of the DSM-5 Yale Food Addiction Scale in a nonclinical sample. The Canadian Journal of Psychiatry, 62(3), 199-210.

Burgess, E. E., Turan, B., Lokken, K. L., Morse, A., \& Boggiano, M. M. (2014). Profiling motives behind hedonic eating. Preliminary validation of the Palatable Eating Motives Scale. Appetite, 72, 66-72. 
Carlson, L., Steward, T., Agüera, Z., Mestre-Bach, G., Magaña, P., Granero, R., ... \& Fernández Aranda, F. (2018). Associations of food addiction and nonsuicidal self-injury among women with an eating disorder: A common strategy for regulating emotions?. European Eating Disorders Review, 26(6), 629-637.

Carr, M. M., Catak, P. D., Pejsa-Reitz, M. C., Saules, K. K., \& Gearhardt, A. N. (2017). Measurement invariance of the Yale Food Addiction Scale 2.0 across gender and racial groups. Psychological Assessment, 29(8), 1044.

Coutinho, J., Ribeiro, E., Ferreirinha, R., \& Dias, P. (2010). Versão portuguesa da Escala de Dificuldades de Regulação Emocional e sua relação com sintomas psicopatológicos. Archives of Clinical Psychiatry, 37(4), 145-151.

Fairburn, C. G., \& Beglin, S. J. (2008). Eating disorder examination questionnaire. Cognitive behavior therapy and eating disorders, $309-313$.

Fawzi, M., \& Fawzi, M. (2018). Validation of an Arabic version of the Yale Food Addiction Scale 2.0. Eastern Mediterranean Health Journal, 24(8).

Field, A. P. (2013). Discovering statistics using SPSS (4th ed.). England: SAGE.

Fornell, C., \& Larcker, D. F. (1981). Evaluating structural equation models with unobservable variables and measurement errors. Journal of Marketing Research, 18(1), 39-50. https://doi.org/doi.org/10.2307/3151312

Gearhardt, A. N., Corbin, W. R., \& Brownell, K. D. (2009). Preliminary validation of the Yale food addiction scale. Appetite, 52(2), 430-436.

Gearhardt, A. N., Corbin, W. R., \& Brownell, K. D. (2016). Development of the Yale Food Addiction Scale Version 2.0. Psychology of Addictive Behaviors, 30(1), 113.

Gearhardt, A. N., Yokum, S., Orr, P. T., Stice, E., Corbin, W. R., \& Brownell, K. D. (2011). Neural correlates of food addiction. Archives of general psychiatry, 68(8), 808-816.

Granero, R., Jiménez-Murcia, S., Gearhardt, A. N., Agüera, Z., Aymamí, N., Gómez-Peña, M., ... \& Riesco, N. (2018). Validation of the Spanish version of the Yale Food Addiction Scale 2.0 (YFAS 2.0) and clinical correlates in a sample of eating disorder, gambling disorder, and healthy control participants.

Frontiers in psychiatry, 9, 208.

Gratz, K. L., \& Roemer, L. (2004). Multidimensional assessment of emotion regulation and dysregulation: Development, factor structure, and initial validation of the difficulties in emotion regulation scale. Journal of psychopathology and behavioral assessment, 26(1), 41-54.

Hasin, D. S., O’Brien, C. P., Auriacombe, M., Borges, G., Bucholz, K., Budney, A., ... \& Schuckit, M. (2013). DSM-5 criteria for substance use disorders: recommendations and rationale. American Journal of Psychiatry, 170(8), 834-851.

Hayes, A. F. (2013). Introduction to mediation, moderation, and conditional process analysis. The Guilford Press.

Horsager, C., Færk, E., Lauritsen, M. B., \& Østergaard, S. D. (2021). Food addiction comorbid to mental disorders: A nationwide survey and register-based study. International Journal of Eating Disorders, 1-16. https://doi.org/10.1002/eat.23472

Jorgensen, T. D., Pornprasertmanit, S., Schoemann, A. M., \& Rosseel, Y. (2018). semTools: Useful tools for structural equation modeling. R package version 0.5-1. Retrieved from https://CRAN.R-project.org/package=semTools.

Khine, M. T., Ota, A., Gearhardt, A. N., Fujisawa, A., Morita, M., Minagawa, A., ... \& Yatsuya, H. (2019). Validation of the japanese version of the yale food addiction scale 2.0 (J-YFAS 2.0). Nutrients, 11(3), 687.

Kim, J. H., Song, J. H., Kim, R., Jang, M. Y., Hong, H. J., Kim, H. J., \& Shin, S. H. (2019). Validity and Reliability of a Korean Version of Yale Food Addiction Scale for Children (YFAS-C). Journal of Korean Academy of Nursing, 49(1), 59-68.

Long, C. G., Blundell, J. E., \& Finlayson, G. (2015). A systematic review of the application and correlates of YFAS-diagnosed 'food addiction'in humans: are eating-related 'addictions' a cause for concern or empty concepts?. Obesity Facts, 8(6), 386-401.

Machado, P. P., Martins, C., Vaz, A. R., Conceição, E., Bastos, A. P., \& Gonçalves, S. (2014). Eating disorder examination questionnaire: psychometric properties and norms for the Portuguese population. European eating disorders review, 22(6), 448-453.

Manzoni, G. M., Rossi, A., Pietrabissa, G., Mannarini, S., Fabbricatore, M., Imperatori, C., Innamorati, M., Gearhardt, A. N., \& Castelnuovo, G. (2020). Structural validity, measurement invariance, reliability and diagnostic accuracy of the Italian version of the Yale Food Addiction Scale 2.0 in patients with severe obesity and the general population. Eating and Weight Disorders - Studies on Anorexia, Bulimia and Obesity, 26(1), 345-366.

https://doi.org/10.1007/s40519-020-00858-y

Meadows, A., Nolan, L. J., \& Higgs, S. (2017). Self-perceived food addiction: Prevalence, predictors, and prognosis. Appetite, 114, $282-298$.

https://doi.org/10.1016/j.appet.2017.03.051

Page $7 / 11$ 
Meule, A., \& Gearhardt, A. N. (2019). Ten years of the Yale Food Addiction Scale: a review of version 2.0. Current Addiction Reports, 6(3), $218-228$.

Meule, A., Müller, A., Gearhardt, A. N., \& Blechert, J. (2017). German version of the Yale Food Addiction Scale 2.0: prevalence and correlates of 'food addiction'in students and obese individuals. Appetite, 115, 54-61.

Orbach, I., \& Mikulincer, M. (1998). The Body Investment Scale: Construction and validation of a body experience scale. Psychological Assessment, 10(4), 415 .

Pedram, P., Wadden, D., Amini, P., Gulliver, W., Randell, E., Cahill, F., ... \& Ji, Y. (2013). Food addiction: its prevalence and significant association with obesity in the general population. PloS one, 8(9).

R Development Core Team (2019). R: A language and environment for statistical computing. R Foundation for Statistical Computing Vienna, Austria: R Foundation for Statistical Computing. Retrieved from http://www.R-project.org/.

Rosseel, Y. (2012). Lavaan: An R package for structural equation modeling. Journal of Statistical Software, 48(2), 1-36. doi: 10.18637/jss. v048.i02.

Rossi, A., Gearhardt, A. N., Castelnuovo, G., \& Mannarini, S. (2020, September 28-29). Different methods of assessment, food addiction, emotional eating, and binge eating behaviors: Comparing the total model effects of sequential mediation analysis [Conference session]. Second Symposium on Psychology-Based Technologies, Naples, Italy.

Sevinçer, G. M., Konuk, N., Bozkurt, S., Saraçlı, Ö., \& Coşkun, H. (2014). Psychometric properties of the Turkish version of the Yale Food Addiction Scale among bariatric surgery patients.

Şanlier, N., Türközü, D., \& Toka, O. (2016). Body Image, Food Addiction, Depression, and Body Mass Index in University Students. Ecology of Food and Nutrition, 55(6), 491-507. https://doi.org/10.1080/03670244.2016.1219951

Sengor, G., \& Gezer, S. (2020). The association between food addiction, disordered eating behaviors and food intake. Revista de Nutrição, 33 , e190039. http://dx.doi.org/10.1590/1678-9865202033e190039

Torres, S., Camacho, M., Costa, P., Ribeiro, G., Santos, O., Vieira, F. M., ... \& Oliveira-Maia, A. J. (2017). Psychometric properties of the Portuguese version of the Yale Food Addiction Scale. Eating and Weight Disorders-Studies on Anorexia, Bulimia and Obesity, 22(2), $259-267$.

Vieira, A. I., Fernandes, J., Machado, P. P. P., \& Gonçalves, S. (2020). The Portuguese version of the body investment scale: psychometric properties and relationships with disordered eating and emotion dysregulation. Journal of Eating Disorders, 8, 24. https://doi.org/10.1186/s40337-020-00302-7

West, S. G., Taylor, A. B., \& Wu, W. (2012). Model Fit and Model Selection in Structural Equation Modeling. In Hoyle, R. H. (Ed.), Handbook of structural equation modeling (pp. 209-231). New York: Guilford press.

Wu, Y. K., Zimmer, C., Munn-Chernoff, M. A., \& Baker, J. H. (2020). Association between food addiction and body dissatisfaction among college students: The mediating role of eating expectancies. Eating Behaviors, 39, 101441. https://doi.org/10.1016/j.eatbeh.2020.101441

\section{Tables}

Table 1 Frequencies and factor loadings of the Yale Food Addiction Scale 2.0 criteria

\begin{tabular}{lcccc}
\hline Criteria & Met criteria & Did not meet criteria & Estimate & SE \\
\hline 1. Substance taken in larger amount and for longer period than intended & $34(11.3)$ & $268(88.7)$ & $.776^{* * *}$ & .02 \\
2. Persistent desire or repeated unsuccessful attempts to quit & $43(14.2)$ & $259(85.8)$ & $.869^{* * *}$ & .02 \\
3. Much time/ activity to obtain, use or recover & $22(7.3)$ & $280(92.7)$ & $.917^{* * *}$ & .01 \\
4. Important social, occupational, or recreational activities given up or reduced & $21(7)$ & $281(93)$ & $.870^{* * *}$ & .01 \\
5. Use continues despite knowledge of adverse consequences (e.g., emotional problems) & $26(8.6)$ & $276(91.4)$ & $.858^{* * *}$ & .02 \\
6. Tolerance & $20(6.6)$ & $282(93.4)$ & $.868^{* * *}$ & .01 \\
7. Characteristic withdrawal symptoms; substance taken to relieve withdrawal & $55(18.2)$ & $247(81.8)$ & $.769^{* * *}$ & .02 \\
8. Continue use despite social or interpersonal problems & $26(8.6)$ & $276(91.4)$ & $.762^{* * *}$ & .02 \\
9. Failure to fulfill major role obligation (e.g., work, school) & $13(4.3)$ & $289(95.7)$ & $.882^{* * *}$ & .01 \\
10. Use physically hazardous situations & $33(10.9)$ & $269(89.1)$ & $.707^{* * *}$ & .02 \\
11. Craving or a strong desire to urge to use & $18(6)$ & $284(94)$ & $.755^{* * *}$ & .01 \\
\hline
\end{tabular}

$* * * p<.001$.

Table 2 Spearman's correlations between YFAS 2.0 symptom count and convergent and divergent measures 


\begin{tabular}{|c|c|c|c|c|c|c|c|c|c|c|c|c|c|c|c|c|c|c|c|}
\hline & 1 & 2 & 3 & 4 & 5 & 6 & 7 & 8 & 9 & 10 & 11 & 12 & 13 & 14 & 15 & 16 & 17 & 18 & 19 \\
\hline $\begin{array}{l}\text { 1.YFAS } 2.0 \\
\text { symptom } \\
\text { count }\end{array}$ & - & & & & & & & & & & & & & & & & & & \\
\hline 2. BMI & $.220^{* * *}$ & - & & & & & & & & & & & & & & & & & \\
\hline $\begin{array}{l}\text { 3. EDE-Q } \\
\text { restraint }\end{array}$ & $.322^{* * *}$ & $.288^{* * *}$ & - & & & & & & & & & & & & & & & & \\
\hline $\begin{array}{l}\text { 4. EDE-Q } \\
\text { eating } \\
\text { concern }\end{array}$ & $.532^{* * *}$ & $.254^{* * *}$ & $.675^{* * *}$ & - & & & & & & & & & & & & & & & \\
\hline $\begin{array}{l}\text { 5. EDE-Q } \\
\text { shape } \\
\text { concern }\end{array}$ & $.476^{* * *}$ & $.288^{* * *}$ & $.648^{* * *}$ & $.700^{* * *}$ & - & & & & & & & & & & & & & & \\
\hline $\begin{array}{l}\text { 6. EDE-Q } \\
\text { weight } \\
\text { concern }\end{array}$ & $.408^{* * *}$ & $.354^{* * *}$ & $.625^{* * *}$ & $.637^{* * *}$ & $.860^{* * *}$ & - & & & & & & & & & & & & & \\
\hline $\begin{array}{l}\text { 7. EDE-Q } \\
\text { total score }\end{array}$ & $.469^{* * *}$ & $.341^{* * *}$ & $.787^{* * *}$ & $.768^{* * *}$ & $.942^{* * *}$ & $.934^{* * *}$ & - & & & & & & & & & & & & \\
\hline $\begin{array}{l}\text { 8. Binge } \\
\text { eating } \\
\text { episodes }\end{array}$ & $.413^{* * *}$ & $.139^{* * *}$ & $.404^{* * *}$ & $.577^{* * *}$ & $.404^{* * *}$ & $.334^{* * *}$ & $.433^{* * *}$ & - & & & & & & & & & & & \\
\hline $\begin{array}{l}\text { 9. BIS Body } \\
\text { image }\end{array}$ & $-.393^{* * *}$ & $-.249^{* * *}$ & $-.423^{* * *}$ & $-.519^{* * *}$ & $-.780^{* * *}$ & $-.691^{* * *}$ & $-.714^{* * *}$ & $-.265^{* * *}$ & - & & & & & & & & & & \\
\hline 10. BIS & $-.253^{* * *}$ & -.056 & -.173 & $-.275^{* * *}$ & $-.270^{* * *}$ & $-.166^{* * *}$ & $-.229^{* * *}$ & $-.181^{* * *}$ & $.411^{* * *}$ & - & & & & & & & & & \\
\hline Body touch & & & & & & & & & & & & & & & & & & & \\
\hline 12. BIS & $-.234^{* * *}$ & -.096 & $-.148^{* * *}$ & $-.229^{* * *}$ & $-.270^{* * *}$ & $-.166^{* * *}$ & $-.177^{* * *}$ & $-.213^{* * *}$ & $.337^{* * *}$ & $.328^{* * *}$ & $.208^{* * *}$ & - & & & & & & & \\
\hline $\begin{array}{l}\text { Body } \\
\text { protection }\end{array}$ & & & & & & & & & & & & & & & & & & & \\
\hline $\begin{array}{l}\text { 13. DERS } \\
\text { Non- } \\
\text { acceptance }\end{array}$ & $.170^{* * *}$ & -.001 & .080 & $.233^{* * *}$ & $.295^{* * *}$ & $.234^{* * *}$ & $.246^{* * *}$ & $.183^{* * *}$ & $-.392^{* * *}$ & $-.218^{* * *}$ & $.138^{* * *}$ & $-.235^{* * *}$ & - & & & & & & \\
\hline 14. DERS & $.239^{* * *}$ & -.002 & $.118^{*}$ & $.279^{* * *}$ & $.299^{* * *}$ & $.206^{* * *}$ & $.249^{* * *}$ & $.255^{* * *}$ & $-.326^{* * *}$ & $-.182^{* * *}$ & -.032 & $-.131^{* * *}$ & $.530^{* * *}$ & - & & & & & \\
\hline Goals & & & & & & & & & & & & & & & & & & & \\
\hline 15. DERS & $.187^{* * *}$ & .094 & .093 & $.240^{* * *}$ & $.311^{* * *}$ & $.238^{* * *}$ & $.258^{* * *}$ & $.187^{* * *}$ & $-.398^{* * *}$ & $-.272^{* * *}$ & $-.283^{* * *}$ & $-.272^{* * *}$ & $.589^{* * *}$ & $.692^{* * *}$ & - & & & & \\
\hline Impulses & & & & & & & & & & & & & & & & & & & \\
\hline $\begin{array}{l}\text { 16. DERS } \\
\text { Strategies }\end{array}$ & $.288^{* * *}$ & .012 & $.156^{* * *}$ & $.306^{* * *}$ & $.386^{* * *}$ & $.317^{* * *}$ & $.348^{* * *}$ & $.226^{* * *}$ & $-.490^{* * *}$ & $-.348^{* * *}$ & $-.193^{* * *}$ & $-.279^{* * *}$ & $.700^{* * *}$ & $.699^{* * *}$ & $.692^{* * *}$ & - & & & \\
\hline $\begin{array}{l}\text { 17. DERS } \\
\text { Awareness }\end{array}$ & $.153^{* * *}$ & $.118^{*}$ & .092 & $.176^{* * *}$ & $.245^{* * *}$ & $.199^{* * *}$ & $.207^{* * *}$ & $.054^{* * *}$ & $-.324^{* * *}$ & $-.305^{* * *}$ & $-.419^{* * *}$ & $-.309^{* * *}$ & $.600^{* * *}$ & $.433^{* *}$ & $.265^{* * *}$ & $.287^{* * *}$ & - & & \\
\hline 18. DERS & $.233^{* * *}$ & $.126^{*}$ & $.196^{* * *}$ & $.306^{* * *}$ & $.386^{* * *}$ & $.317^{* * *}$ & $.348^{* * *}$ & $.203^{* * *}$ & $-.459^{* * *}$ & $-.347^{* * *}$ & $-.270^{* * *}$ & $-.316^{* * *}$ & $.287^{* * *}$ & $.544^{* * *}$ & $.265^{* * *}$ & $.287^{* * *}$ & $.545^{* * *}$ & - & \\
\hline Clarity & & & & & & & & & & & & & & & & & & & \\
\hline $\begin{array}{l}\text { 19. DERS } \\
\text { total }\end{array}$ & $.274^{* * *}$ & .060 & $.151^{* * *}$ & $.401^{* * *}$ & $.305^{* * *}$ & $.328^{* * *}$ & $.339^{* * *}$ & $.248^{* * *}$ & $-.504^{* * *}$ & $-.353^{* * *}$ & $-.249^{* * *}$ & $-.319^{* * *}$ & $.796^{* * *}$ & $.746^{* * *}$ & $.813^{* * *}$ & $.966^{* * *}$ & $.433^{* * *}$ & $.735^{* * *}$ & - \\
\hline
\end{tabular}

Note. YFAS $2.0=$ Yale Food addiction Scale 2.0; EDE-Q = Eating Disorders Examination-Questionnaire; BIS $=$ Body Investment Scale; DERS $=$ Difficulties in Emotion Regulation Scale.

${ }^{*} p<.05 .{ }^{* * *} p<.01$.

Table 3 Comparison of questionnaires according to YFAS 2.0 severity 


\begin{tabular}{|c|c|c|c|c|c|c|c|}
\hline & $\begin{array}{c}\text { 1. No FA } \\
(n=286) \\
M(S D)\end{array}$ & $\begin{array}{l}\text { 2. Mild FA } \\
(n=3) \\
M(S D)\end{array}$ & $\begin{array}{l}\text { 3. Moderate FA } \\
(n=5) \\
M(S D)\end{array}$ & $\begin{array}{l}\text { 4. Severe FA } \\
\begin{array}{l}(n=8) \\
M(S D)\end{array}\end{array}$ & Anova $F$ & $P$ & Pairwise differences ${ }^{a}$ \\
\hline Age & $21.25(3.03)$ & $22.67(3.06)$ & $24.80(6.83)$ & $23.00(5.86)$ & 2.89 & .036 & $1<3$ \\
\hline BMI & 22.34 (4.77) & $20.10(2.64)$ & $28.83(6.70)$ & 24.45 (4.37) & 3.65 & .013 & $1<3$ \\
\hline EDE-Q restraint & $.93(1.26)$ & $3.4(1.26)$ & $2.08(2.09)$ & $2.57(1.62)$ & 8.89 & $<.001$ & $1<2,4$ \\
\hline EDE-Q eating concern & $.47(.74)$ & $2.53(1.86)$ & $2.44(2.23)$ & $3.00(1.15)$ & 41.05 & $<.001$ & $1<2,3,4$ \\
\hline EDE-Q shape concern & $1.78(1.41)$ & $4.63(1.95)$ & $4.83(1.11)$ & $4.89(.77)$ & 23.81 & $<.001$ & $1<2,3,4$ \\
\hline EDE-Q weight concern & $1.86(1.61)$ & $3.67(2.82)$ & $4.70(1.1 .1)$ & $4.81(1.44)$ & 14.91 & $<.001$ & $1<3,4$ \\
\hline EDE-Q total score & $.47(.74)$ & $2.53(1.86)$ & $2.44(2.23)$ & $3.00(1.15)$ & 23.57 & $<.001$ & $1<2,3,4$ \\
\hline Binge eating episodes & $1.65(3.16)$ & $1.67(1.53)$ & $6.00(5.48)$ & $13.63(11.02)$ & 31.96 & $<.001$ & $1<3,4$ \\
\hline BIS Body image & $3.59(.91)$ & $3.00(1.26)$ & $2.33(.91)$ & $1.87(.63)$ & 12.44 & $<.001$ & $1<3,4$ \\
\hline BIS Body touch & $3.61(.75)$ & $3.05(1.21)$ & $3.17(1.12)$ & $3.33(.88)$ & 1.39 & .244 & - \\
\hline BIS Body care & $4.29(.48)$ & $4.06(.19)$ & $3.93(.68)$ & $3.77(.95)$ & 3.79 & .011 & $1<4$ \\
\hline BIS Body protection & $4.16(.56)$ & $4.11(.42)$ & $3.80(.86)$ & $3.52(.62)$ & 3.99 & .008 & $1<4$ \\
\hline DERS Non-acceptance & $13.96(6.39)$ & $10.67(4.51)$ & $20.00(7.17)$ & $19.75(6.92)$ & 3.81 & .011 & $1<4$ \\
\hline DERS Goals & $15.50(4.81)$ & $12.67(2.52)$ & $17.40(2.88)$ & $17.87(4.42)$ & 1.26 & .289 & - \\
\hline DERS Impulses & $12.97(5.43)$ & $11.00(6.93)$ & $17.80(6.93)$ & $15.37(3.92)$ & 1.92 & .127 & - \\
\hline DERS Strategies & $18.51(7.27)$ & $15.00(6.00)$ & $26.20(8.47)$ & $23.87(6.24)$ & 3.46 & .017 & - \\
\hline DERS Awareness & $13.53(4.19)$ & $10.33(4.16)$ & $15.60(2.88)$ & $16.12(4.70)$ & 1.97 & .119 & - \\
\hline DERS Clarity & $11.03(4.06)$ & $9.00(1.73)$ & $14.00(5.66)$ & $14.25(4.33)$ & 2.70 & .046 & - \\
\hline DERS total & $85.62(24.29)$ & 68.66 (21.07) & $111.00(27.23)$ & $107.25(23.61)$ & 4.30 & .005 & $1<4$ \\
\hline
\end{tabular}

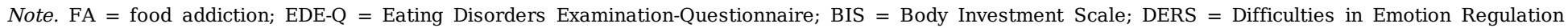
Scale. ${ }^{\mathrm{a}}$ All reported pairwise differences. $p<.05$ (Bonferroni corrected).

Table 4 Moderation Analyses

\begin{tabular}{lcccc}
\hline & $b$ & $S E$ & $t$ & $p$ \\
\hline Constant & .557 & .712 & .782 & .435 \\
Food Addiction & {$[-.845,1.959]$} & & & \\
& -.247 & .424 & -.583 & .561 \\
DERS Goals & {$[-1.081, .587]$} & & & \\
& .022 & .045 & .489 & .625 \\
& {$[-.066, .110]$} & & & \\
Food Addiction x Goals & .075 & .023 & 3.266 & .001 \\
& {$[.030, .121]$} & & & \\
\hline
\end{tabular}

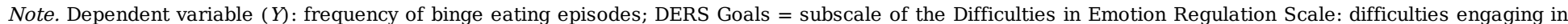
goal-directed behavior when experiencing negative emotions; overall model $R^{2}=.352$.

\section{Figures}

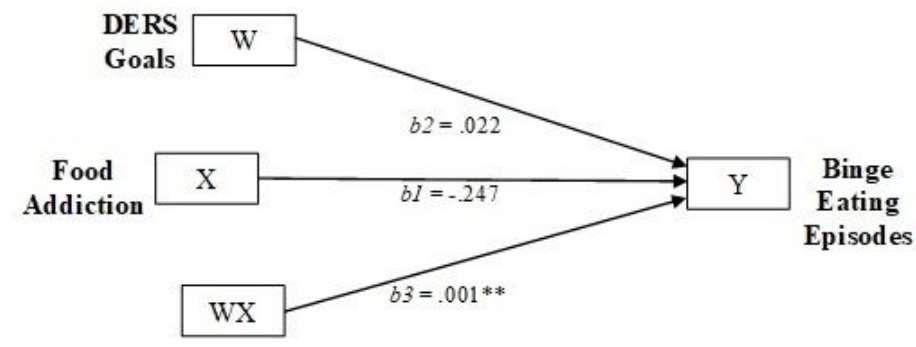

Figure 1 
Moderation of the effect of food addiction on frequency of binge eating episodes by difficulties engaging in goal-directed behavior when experiencing negative emotions Note. DERS Goals = subscale of the Difficulties in Emotion Regulation Scale: difficulties engaging in goal-directed behavior when experiencing negative emotions; $W$ is the moderator; $X$ is the predictor variable; $W X$ is interaction between moderator and predictor; $Y$ is the outcome variable; $b 1$ is the conditional effect of $X$ on $Y$ when $W=0$; $b 2$ is the conditional effect of $W$ on $Y$ when $X=0$; b3 quantifies how the effect of $X$ on $Y$ changes as $\mathrm{W}$ changes by one unit. ** $\mathrm{p}<.01$.

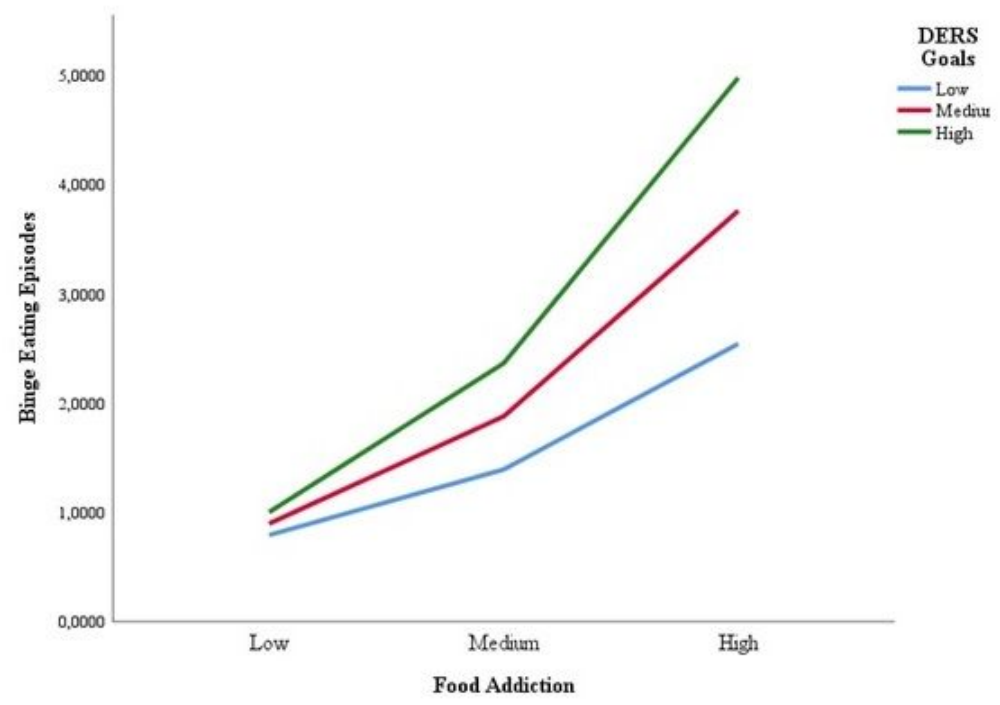

\section{Figure 2}

Visual representation of the moderation of the effect of the food addiction $(X)$ on frequency of binge eating episodes $(Y)$ by low $(\mathrm{W}=10.775)$, medium $(\mathrm{W}=15.549)$ and high $(\mathrm{W}=20.323)$ difficulties engaging in goal-directed behavior when experiencing negative emotions Note. DERS Goals = subscale of the Difficulties in Emotion Regulation Scale: difficulties engaging in goal-directed behavior when experiencing negative emotions. 